\title{
Maternal Vitamin D Status and Risk of Gestational Diabetes: a Meta-Analysis
}

\author{
Lingmin $\mathrm{Hu}^{\mathrm{a}, \mathrm{b}}$ Yue Zhang ${ }^{c} \quad$ Xing Wang ${ }^{\mathrm{b}}$ Lianghui You ${ }^{\mathrm{b}}$ Pengfei Xu ${ }^{\mathrm{b}}$ \\ Xianwei Cui ${ }^{b}$ Lijun Zhu ${ }^{b}$ Chenbo Jib Xirong Guo ${ }^{b} \quad$ Juan Wen ${ }^{b}$
}

aThe Affiliated Changzhou Maternity and Child Health Care Hospital of Nanjing Medical University, Changzhou, ${ }^{b}$ Nanjing Maternity and Child Health Care Institute, Nanjing Maternity and Child Health Care Hospital, Obstetrics and Gynecology Hospital Affiliated to Nanjing Medical University, Nanjing, 'School of Information Management, Nanjing University, Nanjing, China

\section{Key Words}

25-hydroxyvitamin D • Gestational diabetes $•$ Meta-analysis

\begin{abstract}
Background/Aims: Whether maternal vitamin D deficiency is associated with gestational diabetes remains controversial. This meta-analysis aimed to systematically evaluate published evidence on the association between maternal vitamin $D$ status and the risk of gestational diabetes. Methods: We retrieved relevant articles from the PubMed, Medline and Embase databases up to May 2017 for observational studies investigating the association between vitamin $\mathrm{D}$ status and the risk of gestational diabetes. Odds ratios (OR) or risk ratios (RR) from individual studies were pooled using the fixed and random effect models. Results: The meta-analysis of 29 observational studies included 28,982 participants, of which 4,634 were diagnosed with gestational diabetes, and showed that maternal vitamin $D$ insufficiency was associated with a significantly increased risk of gestational diabetes by $39 \%$ (pooled OR = $1.39,95 \% \mathrm{CI}=1.20-1.60)$ with moderate heterogeneity $\left(\mathrm{I}^{2}=50.2 \% ; P=0.001\right)$. Moreover, the $25(\mathrm{OH}) \mathrm{D}$ level was significantly lower in gestational diabetes cases than in controls with a pooled effect of $-4.79 \mathrm{nmol} / \mathrm{L}(95 \% \mathrm{CI}=-6.43,-3.15)$. Significant heterogeneity was also detected $\left(\mathrm{I}^{2}=65.0 \%, P<0.001\right)$. Further subgroup analysis indicated that this association was also evident in most subpopulations. Conclusion: This meta-analysis indicated a significant association between vitamin D insufficiency and increased risk of gestational diabetes. Further well-designed large-scale clinical trials are essential to verify this association.
\end{abstract}

\section{Introduction}

Gestational diabetes, defined by glucose intolerance with the onset or first recognition during pregnancy, is the leading cause of complications associated with childbirth. Its prevalence is increasing globally and has reached nearly 15\%-20\% [1]. Women with

L. Hu and Y. Zhang contributed equally to this work.

Xirong Guo

and Juan Wen

\section{KARGER}


gestational diabetes had an increased risk of developing preeclampsia, dystocia and delivering macrosomia. It also has serious, long-term outcomes for both the mother and their offspring, including a predisposition to obesity, diabetes and metabolic syndrome later in life [2]. Research has shown that advanced maternal age, maternal obesity or overweight and a family history of diabetes could influence the development of gestational diabetes [2]. Whether maternal vitamin D deficiency is associated with gestational diabetes remains unclear. Due to foetal growth needs, inadequate vitamin D intake and limited sunlight exposure, vitamin D deficiency and insufficiency are very common in pregnant women [3]. Multiple studies have reported that maternal vitamin D deficiency during pregnancy appears to be associated with an increased risk of gestational diabetes [4-13]; vitamin D supplementation in gestational diabetes patients had beneficial effects on fasting plasma glucose and serum insulin levels $[14,15]$. Moreover, some studies have shown that vitamin D is required for the normal production and secretion of insulin by the endocrine pancreas [16, 17]. However, controversial results are also abundant, suggesting no significant differences in vitamin $\mathrm{D}$ status between women with gestational diabetes and normal glucose tolerance [16-34]. Thus, to systematically evaluate published evidence on the association between maternal vitamin D status and the risk of gestational diabetes, we conducted an extensive literature search and meta-analysis.

\section{Materials and Methods}

\section{Search Strategy and Selection Criteria}

This meta-analysis was performed and reported according to the Preferred Reporting Items for Systematic Reviews and Meta-Analysis Statement issued in 2009 (Checklist S1) [35]. Institutional Review Board approval (2016(8)) was obtained from Nanjing Maternity and Child Health Care Institute.

We retrieved relevant articles in the PubMed, Medline and Embase databases up to May 2017 using the following search parameters: any observational study investigating the association between vitamin $\mathrm{D}$ status and the risk of gestational diabetes, with "vitamin D" OR "25(OH)D" OR "25-hydroxyvitamin D" OR "cholecalciferol" AND "gestational diabetes" as the search terms. Furthermore, we reviewed the reference lists in the retrieved articles and review articles to identify additional studies that may be eligible for inclusion in this analysis.

Studies were included in our pooled analysis if: 1) the study population was pregnant women; 2) gestational diabetes was the outcome and the control group consisted of women with normal glucose tolerance; 3) the associations between vitamin D status and risk of gestational diabetes were evaluated; 4) an effect estimate [odds ratios (OR) or risk ratios (RR)] with 95\% confidence intervals (CI) for comparisons of vitamin D insufficiency and sufficiency was provided or could be calculated; 5) the article was published in English (Fig. 1). We excluded studies that were case reports or not published as full reports, studies where the vitamin D level tested during or after delivery, and studies without control subjects or with an inappropriate comparison group.

\section{Independent Assessment}

Two investigators independently searched and reviewed all the retrieved articles using a standardized approach, and data were extracted via a standardized data extraction form and checked by other investigators. The two investigators were blinded to identify information from each study and judged the inclusion and exclusion criteria of the study.

The data collected included the first author's last name, publication year, study location, study design, number of participants and gestational diabetes cases, gestational diabetes criteria, assessment of vitamin $D$, mean (SD) or median (IQR) concentrations of 25(OH)D, vitamin D cut-off value, adjustment for confounders, the effect estimate with $95 \% \mathrm{CI}$, and other related information. As there were different vitamin D cut-off values among different studies, we defined the maternal vitamin D status in this meta-analysis based on any levels reported as vitamin D deficiency or insufficiency from these studies (range from 25 to $75 \mathrm{nmol} / \mathrm{L}$ ). If any of these data were missing, the respective authors were contacted for relevant information. The 
concordance rate between the two investigators was $98.7 \%$. Any discrepancies were resolved by consensus with group discussion.

\section{Statistical Analysis}

The effect estimate (OR or RR) with 95\%CI in each study was used to examine the association between vitamin $\mathrm{D}$ status and the risk of gestational diabetes. If the adjusted effect estimate was not given, a crude effect estimate was used. If the OR with $95 \%$ CI was not provided, the data were calculated by a $2 \times 2$ table. Forest plots were used to visually assess pooled estimates and corresponding 95\% CIs. Statistical heterogeneity among studies was tested using the $\chi^{2}$ test, $I^{2}$ statistics, and $P$ values [36]. In the presence of non-significant heterogeneity $(P>0.10)$, a fixed-effects model was used to calculate the pooled effect size, otherwise, a random-effects model was applied. Sensitivity analyses were performed by removing individual studies to evaluate the influence of the included study in our meta-analysis. Subgroup analyses were also conducted for the subgroups based on population, study design, number of participants, gestational diabetes criteria, assessment of vitamin D, trimester, significant difference in 25(OH)D between groups, cut-off value, and adjustment for confounders. Potent publication bias was evaluated by using funnel plots, Begg's test and Egger's test [37]. A $P$ value less than 0.10 was considered to indicate significant publication bias. All statistical analyses were two-sided and performed using Stata software (version 11.0; Stata Corp).

\section{Results}

The flow chart of the study selection is described in Fig. 1. The relevant 129 articles were identified in our initial search, of which 79 were excluded after screening titles and/or abstracts. A total of 50 potentially eligible studies were selected. After detailed evaluations, 29 observational studies were included for the final pooled analysis [4-13, 16-34].

A summary of the included studies is presented in Table 1. The earliest study was published in 2008 and the latest in 2017, but more than half of the studies were published within the past 5 years. The 29 included studies consisted of 13 cohort studies, 8 nested casecontrol studies, 5 case-control studies, and 3 cross-sectional studies. In total, these studies

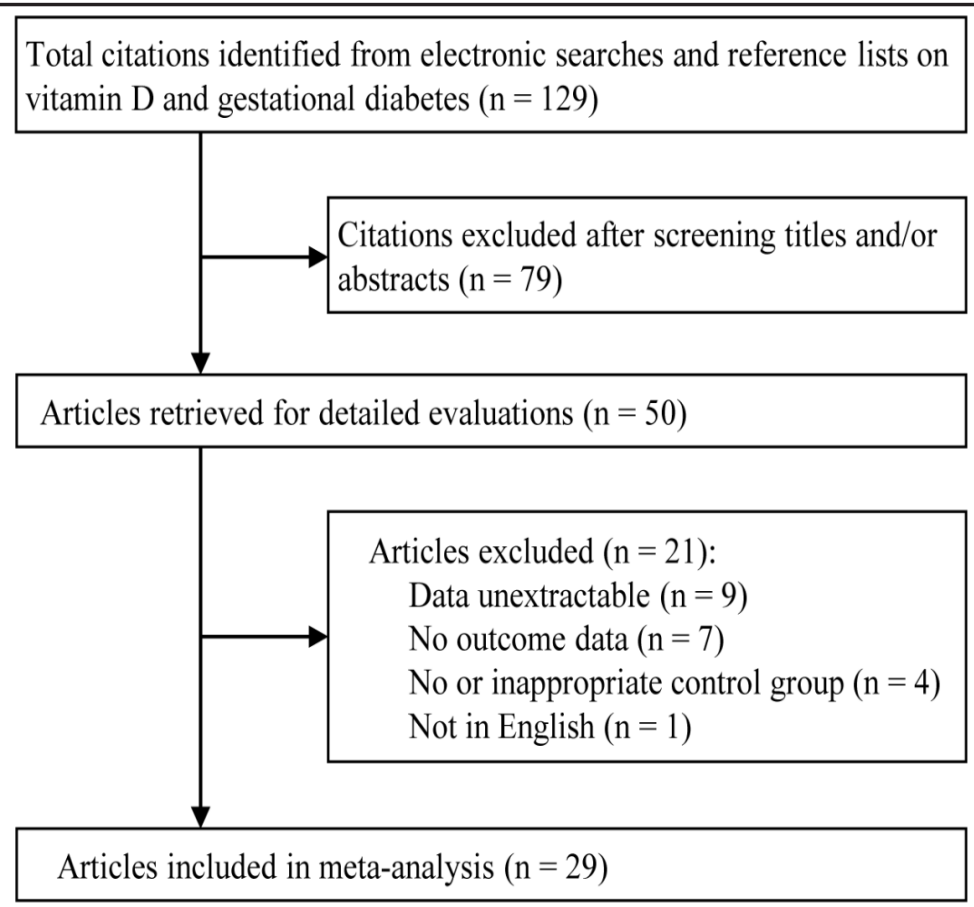

Fig. 1. Flow chart of study selection in review of the association of maternal serum 25-hydroxyvitamin D status with gestational diabetes. 

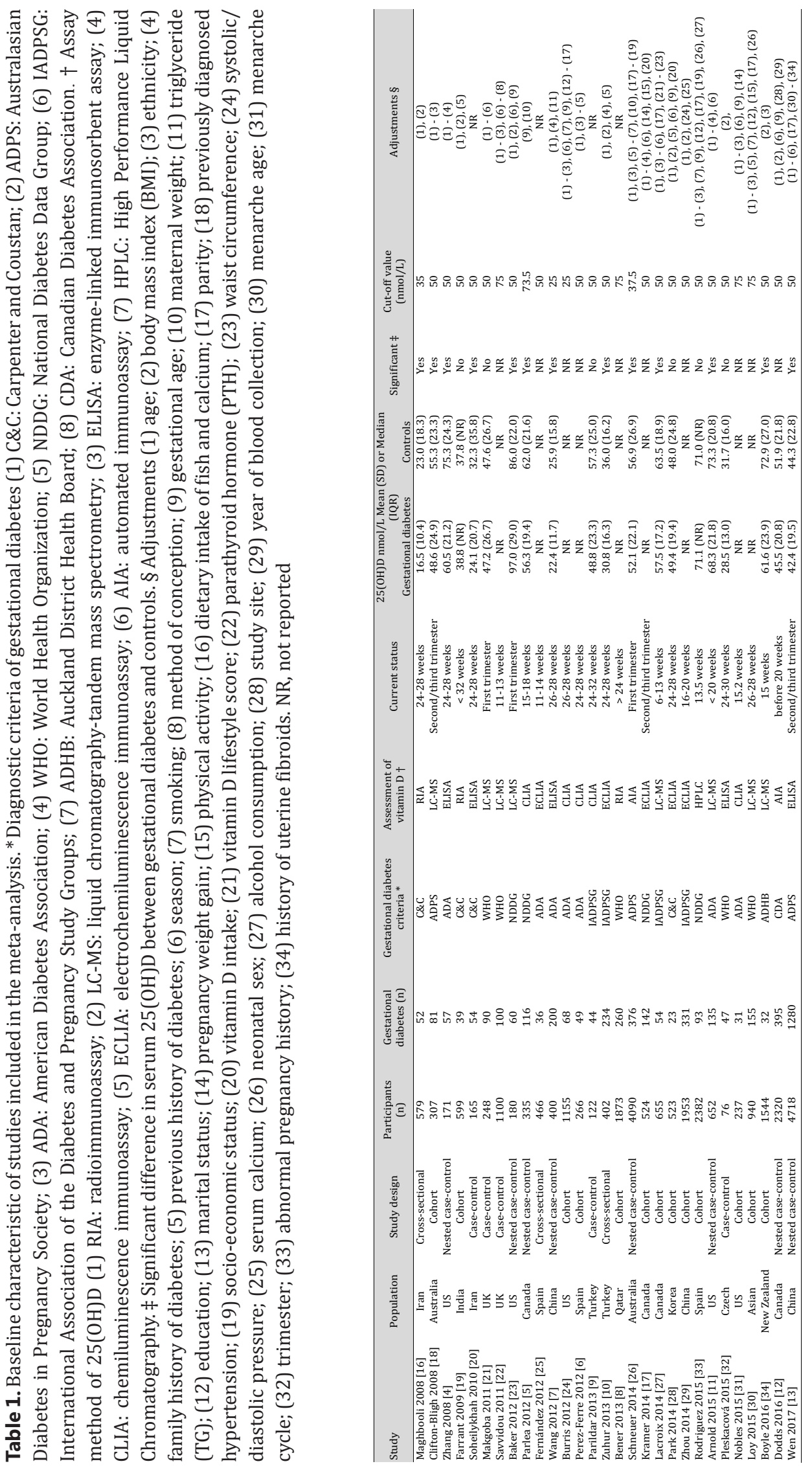
involved 28, 982 participants and 4, 634 gestational diabetes cases with various ethnicities. The sample size of the participants in each study ranged from 76 to 4718 . Only 9 of the 29 studies had more than 1, 000 participants, and the majority of studies (72\%) were conducted in developed countries. Furthermore, the gestational diabetes criteria, assessment of vitamin D and the current status of pregnant women in these studies were different. The 25(OH)D levels in gestational diabetes cases in each study showed a wide variety, ranging from 16.5 to $97.0 \mathrm{nmol} / \mathrm{L}$ with a median of $48.7 \mathrm{nmol} / \mathrm{L}$. Thirteen studies found a significant difference in maternal 25(OH)D between gestational diabetes cases and controls. For the cut-off value, the included studies primarily used $50 \mathrm{nmol} / \mathrm{L}$. Twenty studies used $50 \mathrm{nmol} / \mathrm{L}, 5$ studies used 73.5-75 nmol/L, and the remaining 4 studies used $25-37.5 \mathrm{nmol} / \mathrm{L}$ as the cut-off value. A few of the studies matched the cases and controls, but most of the studies (86\%) adjusted for confounding factors, such as age, body mass index (BMI), ethnicity, season, gestational age, pregnancy weight gain, physical activity, parity, socioeconomic status, vitamin D intake, alcohol consumption, etc. in different combinations.

Overall, 29 studies were

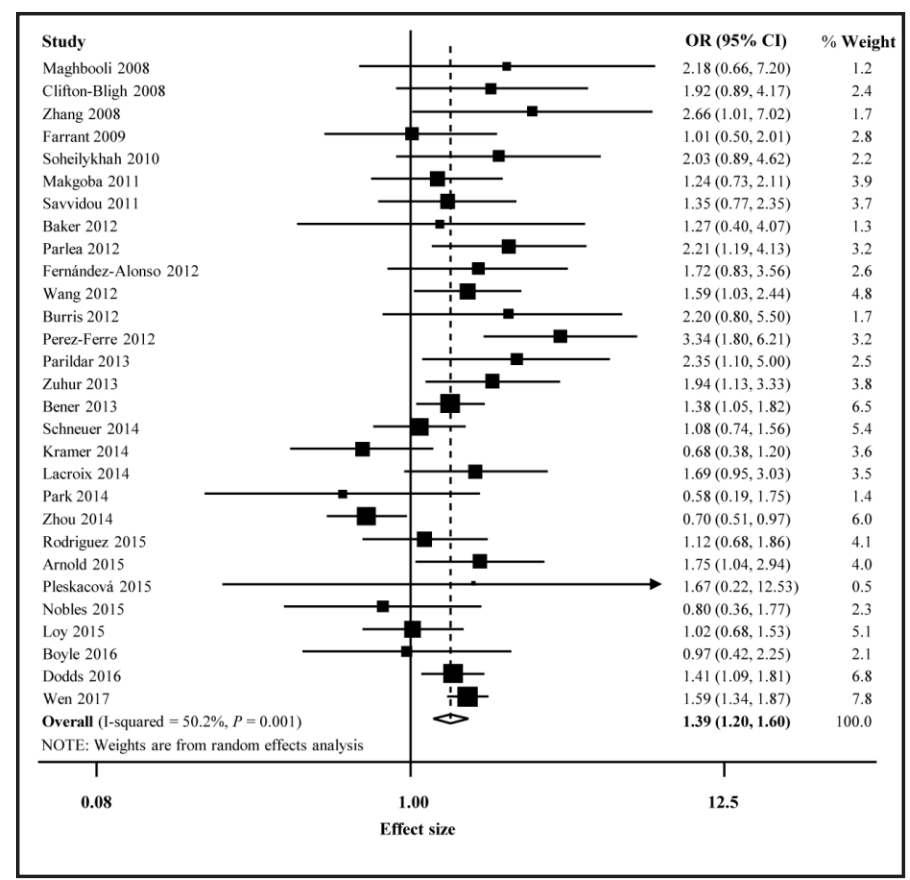

Fig. 2. Meta-analysis of the association between vitamin D insufficiency and risk of gestational diabetes.

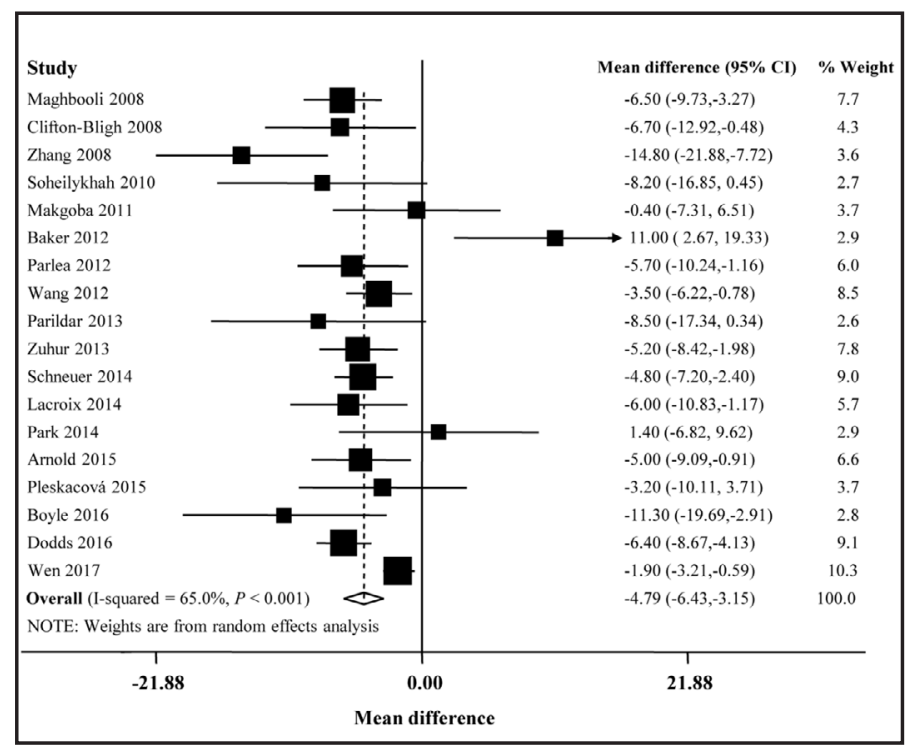

Fig. 3. Meta-analysis of the association between 25(OH)D level and gestational diabetes. used for the meta-analysis to determine the association between vitamin D status and the risk of gestational diabetes (Fig. 2). Ten studies showed an increased risk of gestational diabetes for vitamin D insufficiency, eighteen studies showed no significant association between the two, and only one study showed a reduced risk of gestational diabetes for vitamin D insufficiency. The summary OR showed that maternal vitamin D insufficiency was associated with a significantly increased risk of gestational diabetes by $39 \%$ (pooled $\mathrm{OR}=1.39,95 \% \mathrm{CI}=1.20-1.60$ ), and moderate heterogeneity was detected across the included studies $\left(\mathrm{I}^{2}=50.2 \% ; P=0.001\right)$. The individual risk estimate for these studies ranged from 0.58 to 3.34 . We also conducted a sensitivity analysis to 
Fig. 4. Subgroup meta-analysis of the association between vitamin D insufficiency and risk of gestational diabetes. * Diagnostic criteria of gestational diabetes (1) ADA: American Diabetes Association; (2) ADPS: Australasian Diabetes in Pregnancy Society; (3) WHO: World Health Organization. † Assay method of $25(\mathrm{OH})$ D (1) AIA: automated immunoassay; (2) CLIA: chemiluminescence immunoassay; (3) ECLIA: electrochemiluminescence immunoassay;

RIA: radioimmunoassay; (5) ELISA: enzymelinked immunosorbent assay; (6) LCMS: liquid chromatography-tandem mass spectrometry. $\neq$ Significant difference in serum 25(OH)D between gestational diabetes and controls. § P for heterogeneity among studies.

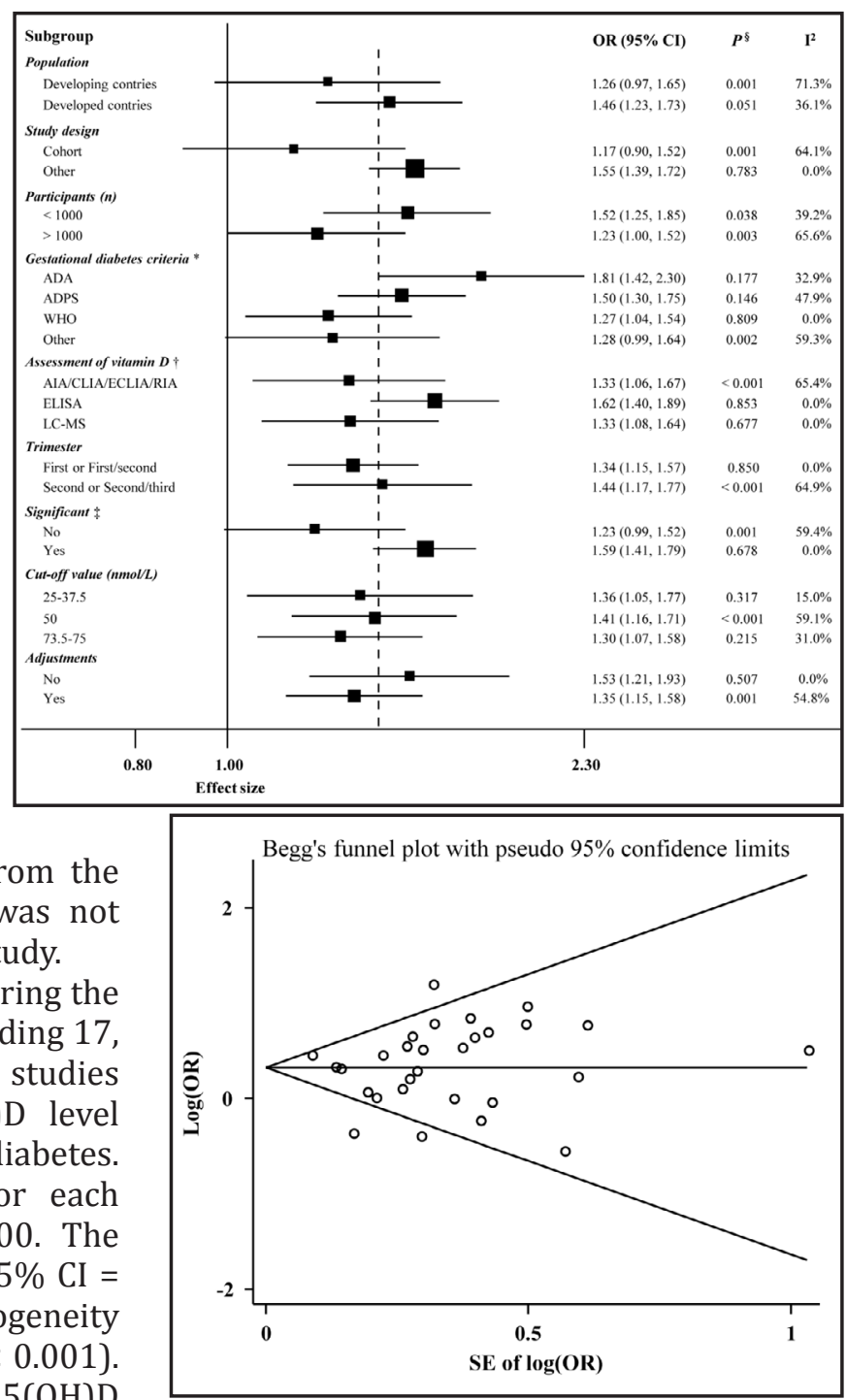

Fig. 5. Funnel plot of $\log (\mathrm{OR})$ against $\mathrm{SE}$ of $\log (\mathrm{OR})$ for all studies. diabetes cases than in controls and demonstrated that vitamin D insufficiency was significantly associated with an increased risk of gestational diabetes.

We also stratified our analysis based on population, study design, number of participants, gestational diabetes criteria, assessment of vitamin D, trimester, significant difference in 25(OH)D between groups, cut-off value, and adjustment for confounders to minimize heterogeneity among the included studies (Fig. 4). In our results, the ORs of the studies conducted in developed countries (vs. developing countries); with a sample size less than 1, 000 (vs. sample size greater than 1,000); and adjusted for confounders (vs. non-adjustments) were larger, but statistical significance for heterogeneity was not reached (heterogeneity test: all $P>0.100)$. The OR of the cohort studies (1.17) was significantly smaller than that of studies with other study designs (1.55) (heterogeneity test: $P=0.051$ ); and the OR of studies with a significant difference in serum 25(OH)D between gestational diabetes and controls (1.59) was significantly larger than that of studies with non-significant difference (1.23) (heterogeneity test: $P=0.039$ ). In addition, subgroup analysis by assessment of vitamin $\mathrm{D}$, trimester, cut-off value, and adjustment for confounders were all significant (Fig. 4). 


\section{Cellular Physiology Cell Physiol Biochem 2018;45:291-300

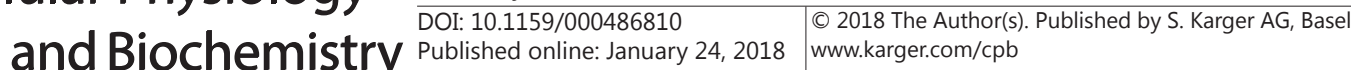 \\ Hu et al.: Maternal 25(OH)D and Gestational Diabetes}

We then evaluated publication bias by using funnel plots, Begg's test and Egger's test (Fig. 5). The $P$ values of the Begg's and Egger's tests were 0.499 and 0.769 , respectively, revealing no obvious publication bias in our analysis.

\section{Discussion}

This report reviewed and pooled epidemiological data assessing the association between vitamin $\mathrm{D}$ status and the risk of gestational diabetes. This comprehensive quantitative metaanalysis included 28, 982 participants and 4,634 gestational diabetes cases with various ethnicities. Our results suggested that maternal vitamin D insufficiency might play an important role in the risk of gestational diabetes, and that the 25(OH)D level of gestational diabetes cases was decreased by $4.79 \mathrm{nmol} / \mathrm{L}$ compared to the control group.

Several previous meta-analyses have suggested that vitamin D insufficiency was associated with an increased risk of gestational diabetes [38-41]. However, these previous studies did not include several important observational studies. Furthermore, the studies did not evaluate the association between the serum 25(OH)D level and gestational diabetes, nor did they evaluate the association in specific subpopulations. Therefore, we conducted an updated meta-analysis including all of the important observational studies to evaluate the association between vitamin $\mathrm{D}$ deficiency and the risk of gestational diabetes, the association between the serum 25(OH)D level and gestational diabetes, as well as subgroup analysis in various subgroups. The subgroup analysis that was performed to reduce selection bias and increase the generalizability of the present results to other populations is the main strength of this study.

There was considerable heterogeneity among the 29 included studies, which was not surprising given the different ethnicities, study designs, number of participants, gestational diabetes criteria, assessment of vitamin D, trimester, cut-off values, and adjustments for confounders. Subgroup analysis suggested that maternal vitamin D insufficiency was associated with increased risk of gestational diabetes only if the following parameters were met: the study was conducted in developed countries, cohort design was not used in the study, criteria from the American Diabetes Association (ADA), Australasian Diabetes in Pregnancy Society (ADPS) or World Health Organization (WHO) was used for the gestational diabetes diagnosis, and a significant difference in serum 25(OH)D was observed between gestational diabetes cases and controls. According to the number of participants, we divided the publications into studies with sample sizes less than 1,000 and greater than 1,000. The OR of the group with a sample size less than 1,000 was significantly larger than that of the group with a sample size of greater than 1,000. Because larger studies are more representative and have less bias, the results of the studies with a larger sample size tended to be more reliable. In addition to vitamin $\mathrm{D}$, there are also many competing risks that could increase the rate of gestational diabetes, including age, BMI, ethnicity, pregnancy weight gain, physical activity, socio-economic status, alcohol consumption, family history of diabetes, previous history of diabetes, etc. Thus, the overall effect decreased after adjustment for some variables.

The observed association between maternal vitamin D insufficiency and increased risk of gestational diabetes is biologically plausible. Alvarez et al. summarized the potential influence of vitamin D on glucose homeostasis [42], including the presence of specific vitamin D receptors (VDRs) on pancreatic $\beta$-cells and skeletal muscle, the presence of a vitamin $D$ response element in the human insulin gene promoter, and the expression of the 1- $\alpha$-hydroxylase enzyme in pancreatic $\beta$-cells which catalyzes the conversion of $25(\mathrm{OH}) \mathrm{D}$ to 1 , 25 -dihydroxyvitamin $\mathrm{D}(1,25(\mathrm{OH}) 2 \mathrm{D})$. In addition, animal and in vitro studies also provided compelling evidence that vitamin D may play a functional role in the preservation of glucose tolerance through its effects on insulin secretion and insulin sensitivity. However, it remains unclear whether the measurement of vitamin D levels and vitamin D supplementation during pregnancy should be recommended. Furthermore, the definition of vitamin D insufficiency 


\section{Cellular Physiology Cell Physiol Biochem 2018;45:291-300

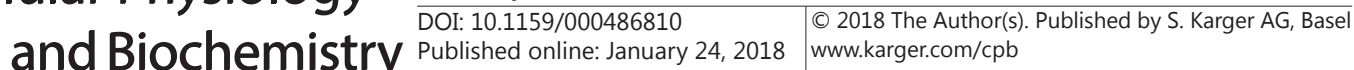 \\ Hu et al.: Maternal 25(OH)D and Gestational Diabetes}

is still not globally uniform. Additional well-designed large-scale clinical trials are needed to verify the association between vitamin D status and the risk of gestational diabetes.

This meta-analysis has several limitations. First, the diagnostic criteria for gestational diabetes, the assay method for 25(OH)D, and the vitamin D cut-off values differed between studies. Further, potential confounding factors in several studies could not be adjusted for, and the adjusted models across the studies differed. Moreover, the long-term risk of adverse outcomes for mother and their offspring as well as data on sunlight exposure were not available and had not been reported by the included studies. Furthermore, the cause of the association between vitamin D insufficiency and the risk of gestational diabetes remains unclear due to the varied study designs across the included studies. Further well-designed randomized controlled trials are needed to evaluate this association and to determine the explicit effect of vitamin D supplementation on the prevention of gestational diabetes.

\section{Acknowledgements}

This work was supported in part by the National Key Basic Research Program of China (2013CB530604), the National Natural Science Foundation of China $(81600685,81402147)$, the Natural Science Foundation of Jiangsu Province (BK20160141), the Medical Science and technology development Foundation of Nanjing Department of Health (YKK16201), the Science and Technology Development Fund of the Nanjing Medical University (2015NJMUZD062), Changzhou Natural Science Foundation (CJ20140021) and Jiangsu Provincial Medical Youth Talent (QNRC2016304). J.W and X.G designed the study. L.H, Y.Y, X.W, L.Y, P.X, X.C and L.Z collected the data. J.W, C.J and X.G conducted the statistical analysis and interpretation. L.H and Y.Y wrote the report. J.W and X.G revised the report. All the authors reviewed the report and approved the final version.

\section{Disclosure Statement}

There are no relevant conflicts of interest to disclose.

\section{References}

1 American Diabetes A: Standards of medical care in diabetes--2014. Diabetes Care 2014;37:S14-80.

2 Reece EA, Leguizamon G, Wiznitzer A: Gestational diabetes: the need for a common ground. Lancet 2009;373:1789-1797.

- 3 Cashman KD, Dowling KG, Skrabakova Z, Gonzalez-Gross M, Valtuena J, De Henauw S, Moreno L, Damsgaard CT, Michaelsen KF, Molgaard C, Jorde R, Grimnes G, Moschonis G, Mavrogianni C, Manios Y, Thamm M, Mensink GB, Rabenberg M, Busch MA, Cox L, Meadows S, Goldberg G, Prentice A, Dekker JM, Nijpels G, Pilz S, Swart KM, van Schoor NM, Lips P, Eiriksdottir G, Gudnason V, Cotch MF, Koskinen S, Lamberg-Allardt C, Durazo-Arvizu RA, Sempos CT, Kiely M: Vitamin D deficiency in Europe: pandemic? Am J Clin Nutr 2016;103:1033-1044.

- Z Zhang C, Qiu C, Hu FB, David RM, van Dam RM, Bralley A, Williams MA: Maternal plasma 25-hydroxyvitamin D concentrations and the risk for gestational diabetes mellitus. PLoS One 2008;3:e3753.

5 Parlea L, Bromberg IL, Feig DS, Vieth R, Merman E, Lipscombe LL: Association between serum 25-hydroxyvitamin D in early pregnancy and risk of gestational diabetes mellitus. Diabet Med 2012;29:e25-32.

6 Perez-Ferre N, Torrejon MJ, Fuentes M, Fernandez MD, Ramos A, Bordiu E, del Valle L, Rubio MA, Bedia AR, Montanez C, Calle-Pascual AL: Association of low serum 25-hydroxyvitamin D levels in pregnancy with glucose homeostasis and obstetric and newborn outcomes. Endocr Pract 2012;18:676-684. 


\section{Cellular Physiology Cell Physiol Biochem 2018;45:291-300 \begin{tabular}{ll|l} 
and Biochemistry Published onlIne: January 24, 2018 & $\begin{array}{l}\text { (C) } 2018 \text { The Author(s). Published by S. Karger AG, Basel } \\
\text { www.karger.com/cpb }\end{array}$ \\
\hline
\end{tabular}}

Hu et al.: Maternal 25(OH)D and Gestational Diabetes

7 Wang O, Nie M, Hu YY, Zhang K, Li W, Ping F, Liu JT, Chen LM, Xing XP: Association between vitamin D insufficiency and the risk for gestational diabetes mellitus in pregnant Chinese women. Biomed Environ Sci 2012;25:399-406.

-8 Bener A, Al-Hamaq AO, Saleh NM: Association between vitamin D insufficiency and adverse pregnancy outcome: global comparisons. Int J Womens Health 2013;5:523-531.

-9 Parildar H, Dogruk Unal A, Aksan Desteli G, Cigerli O, Guvener Demirag N: Frequency of Vitamin D deficiency in pregnant diabetics at Baskent University Hospital, Istanbul. Pak J Med Sci 2013;29:15-20.

10 Zuhur SS, Erol RS, Kuzu I, Altuntas Y: The relationship between low maternal serum 25-hydroxyvitamin D levels and gestational diabetes mellitus according to the severity of 25-hydroxyvitamin D deficiency. Clinics 2013;68:658-664.

11 Arnold DL, Enquobahrie DA, Qiu C, Huang J, Grote N, VanderStoep A, Williams MA: Early pregnancy maternal vitamin D concentrations and risk of gestational diabetes mellitus. Paediatr Perinat Epidemiol 2015;29:200-210.

$>12$ Dodds L, Woolcott CG, Weiler H, Spencer A, Forest JC, Armson BA, Giguere Y: Vitamin D Status and Gestational Diabetes: Effect of Smoking Status during Pregnancy. Paediatr Perinat Epidemiol 2016;30:229237.

13 Wen J, Hong Q, Zhu L, Xu P, Fu Z, Cui X, You L, Wang X, Wu T, Ding H, Dai Y, Ji C, Guo X: Association of maternal serum 25-hydroxyvitamin D concentrations in second and third trimester with risk of gestational diabetes and other pregnancy outcomes. Int J Obes (Lond) 2017;41:489-496.

14 Shahgheibi S, Farhadifar F, Pouya B: The effect of vitamin D supplementation on gestational diabetes in high-risk women: Results from a randomized placebo-controlled trial. J Res Med Sci 2016;21:2.

15 Jamilian M, Samimi M, Ebrahimi FA, Hashemi T, Taghizadeh M, Razavi M, Sanami M, Asemi Z: The effects of vitamin D and omega-3 fatty acid co-supplementation on glycemic control and lipid concentrations in patients with gestational diabetes. J Clin Lipidol 2017;11:459-468.

-16 Maghbooli Z, Hossein-Nezhad A, Karimi F, Shafaei AR, Larijani B: Correlation between vitamin D3 deficiency and insulin resistance in pregnancy. Diabetes Metab Res Rev 2008;24:27-32.

-17 Kramer CK, Swaminathan B, Hanley AJ, Connelly PW, Sermer M, Zinman B, Retnakaran R: Vitamin D and parathyroid hormone status in pregnancy: effect on insulin sensitivity, beta-cell function, and gestational diabetes mellitus. J Clin Endocrinol Metab 2014;99:4506-4513.

18 Clifton-Bligh RJ, McElduff P, McElduff A: Maternal vitamin D deficiency, ethnicity and gestational diabetes. Diabet Med 2008;25:678-684.

19 Farrant HJ, Krishnaveni GV, Hill JC, Boucher BJ, Fisher DJ, Noonan K, Osmond C, Veena SR, Fall CH: Vitamin $\mathrm{D}$ insufficiency is common in Indian mothers but is not associated with gestational diabetes or variation in newborn size. Eur J Clin Nutr 2009;63:646-652.

20 Soheilykhah S, Mojibian M, Rashidi M, Rahimi-Saghand S, Jafari F: Maternal vitamin D status in gestational diabetes mellitus. Nutr Clin Pract 2010;25:524-527.

21 Makgoba M, Nelson SM, Savvidou M, Messow CM, Nicolaides K, Sattar N: First-trimester circulating 25-hydroxyvitamin D levels and development of gestational diabetes mellitus. Diabetes Care 2011;34:1091-1093.

-22 Savvidou MD, Akolekar R, Samaha RB, Masconi AP, Nicolaides KH: Maternal serum 25-hydroxyvitamin D levels at $11(+0)-13(+6)$ weeks in pregnant women with diabetes mellitus and in those with macrosomic neonates. BJOG 2011;118:951-955.

-23 Baker AM, Haeri S, Camargo CA Jr, Stuebe AM, Boggess KA: First-trimester maternal vitamin D status and risk for gestational diabetes (GDM) a nested case-control study. Diabetes Metab Res Rev 2012;28:164-168.

-24 Burris HH, Rifas-Shiman SL, Kleinman K, Litonjua AA, Huh SY, Rich-Edwards JW, Camargo CA, Jr., Gillman MW: Vitamin D deficiency in pregnancy and gestational diabetes mellitus. Am J Obstet Gynecol 2012;207:182 e181-188.

25 Fernandez-Alonso AM, Dionis-Sanchez EC, Chedraui P, Gonzalez-Salmeron MD, Perez-Lopez FR, Spanish Vitamin D, Women's Health Research G: First-trimester maternal serum 25-hydroxyvitamin D(3) status and pregnancy outcome. Int J Gynaecol Obstet 2012;116:6-9.

-26 Schneuer FJ, Roberts CL, Guilbert C, Simpson JM, Algert CS, Khambalia AZ, Tasevski V, Ashton AW, Morris JM, Nassar N: Effects of maternal serum 25-hydroxyvitamin D concentrations in the first trimester on subsequent pregnancy outcomes in an Australian population. Am J Clin Nutr 2013;99:287-295. 


\section{Cellular Physiology Cell Physiol Biochem 2018;45:291-300 \begin{tabular}{ll|l} 
DOI: 10.1159/000486810 & Ond Biochemistry Publisned onine: January 24, 2018 & $\begin{array}{l}\text { O 2018 The Author(s). Published by S. Karger AG, Basel } \\
\text { www.karger.com/cpb }\end{array}$
\end{tabular}}

Hu et al.: Maternal 25(OH)D and Gestational Diabetes

27 Lacroix M, Battista MC, Doyon M, Houde G, Menard J, Ardilouze JL, Hivert MF, Perron P: Lower vitamin D levels at first trimester are associated with higher risk of developing gestational diabetes mellitus. Acta Diabetol 2014;51:609-616.

-28 Park S, Yoon HK, Ryu HM, Han YJ, Lee SW, Park BK, Park SY, Yim CH, Kim SH: Maternal vitamin D deficiency in early pregnancy is not associated with gestational diabetes mellitus development or pregnancy outcomes in Korean pregnant women in a prospective study. J Nutr Sci Vitaminol (Tokyo) 2014;60:269275.

29 Zhou J, Su L, Liu M, Liu Y, Cao X, Wang Z, Xiao H: Associations between 25-hydroxyvitamin D levels and pregnancy outcomes: a prospective observational study in southern China. Eur J Clin Nutr 2014;68:925930.

30 Loy SL, Lek N, Yap F, Soh SE, Padmapriya N, Tan KH, Biswas A, Yeo GS, Kwek K, Gluckman PD, Godfrey KM, Saw SM, Muller-Riemenschneider F, Chong YS, Chong MF, Chan JK, Growing Up in Singapore Towards Healthy Outcomes study g: Association of Maternal Vitamin D Status with Glucose Tolerance and Caesarean Section in a Multi-Ethnic Asian Cohort: The Growing Up in Singapore Towards Healthy Outcomes Study. PLoS One 2015;10:e0142239.

31 Nobles CJ, Markenson G, Chasan-Taber L: Early pregnancy vitamin D status and risk for adverse maternal and infant outcomes in a bi-ethnic cohort: the Behaviors Affecting Baby and You (B.A.B.Y.) Study. Br J Nutr 2015;114:2116-2128.

-32 Pleskačová A, Bartáková V, Pácal L, Kuricová K, Bělobrádková J, Tomandl J, Kaňková K: Vitamin D Status in Women with Gestational Diabetes Mellitus during Pregnancy and Postpartum. BioMed Research International 2015;2015:1-7.

-33 Rodriguez A, Garcia-Esteban R, Basterretxea M, Lertxundi A, Rodriguez-Bernal C, Iniguez C, RodriguezDehli C, Tardon A, Espada M, Sunyer J, Morales E: Associations of maternal circulating 25-hydroxyvitamin D3 concentration with pregnancy and birth outcomes. BJOG 2015;122:1695-1704.

-34 Boyle VT, Thorstensen EB, Mourath D, Jones MB, McCowan LM, Kenny LC, Baker PN: The relationship between 25-hydroxyvitamin D concentration in early pregnancy and pregnancy outcomes in a large, prospective cohort. Br J Nutr 2016;116:1409-1415.

-35 Moher D, Liberati A, Tetzlaff J, Altman DG, Group P: Preferred reporting items for systematic reviews and meta-analyses: the PRISMA statement. PLoS Med 2009;6:e1000097.

-36 Higgins JP, Thompson SG: Quantifying heterogeneity in a meta-analysis. Stat Med 2002;21:1539-1558.

37 Sterne JA, Egger M: Funnel plots for detecting bias in meta-analysis: guidelines on choice of axis. J Clin Epidemiol 2001;54:1046-1055.

-38 Lu M, Xu Y, Lv L, Zhang M: Association between vitamin D status and the risk of gestational diabetes mellitus: a meta-analysis. Arch Gynecol Obstet 2016;293:959-966.

-39 Zhang MX, Pan GT, Guo JF, Li BY, Qin LQ, Zhang ZL: Vitamin D Deficiency Increases the Risk of Gestational Diabetes Mellitus: A Meta-Analysis of Observational Studies. Nutrients 2015;7:8366-8375.

-40 Poel YH, Hummel P, Lips P, Stam F, van der Ploeg T, Simsek S: Vitamin D and gestational diabetes: a systematic review and meta-analysis. Eur J Intern Med 2012;23:465-469.

-41 Aghajafari F, Nagulesapillai T, Ronksley PE, Tough SC, O’Beirne M, Rabi DM: Association between maternal serum 25-hydroxyvitamin D level and pregnancy and neonatal outcomes: systematic review and metaanalysis of observational studies. BMJ 2013;346:f1169.

42 Alvarez JA, Ashraf A: Role of vitamin d in insulin secretion and insulin sensitivity for glucose homeostasis. Int J Endocrinol 2010;2010:351385. 